\title{
Assessment of the patient
}

A relevant history should be taken with particular reference to:

Where?-Site of initial lesion(s) and subsequent changes in distribution.

When?-Have there been any previous episodes? If so, note the duration of these and the present condition.

Progress?-Is it getting better or worse? Does it itch?

Treatment? - Treatment to dateboth by prescription and by home remedies.

What else?-Are there any associated conditions? Ask about any medication and drugs, whether prescribed or bought "over the counter."
We now come to the matter of using these basic concepts in the diagnosis of lesions in practice. The next four articles deal with two common skin diseases-psoriasis, which affects $1-2 \%$ of the population, and eczema, an even more common complaint. Both are rashes with epidermal changes. The difficulty arises with the unusual lesion: Is it a rarity or a variation of a common disease? What should make us consider further investigation? Is it safe to wait and see if it resolves or persists? The usual clinical presentations of psoriasis and eczema are set out and used as a basis for comparison with variations of the usual pattern and other skin conditions.

Dr Paul K Buxton, FRCPED, FRCPC, is consultant dermatologist, Royal Infirmary, Edinburgh, and Fife Health Board.

\section{Basic Molecular and Cell Biology}

\section{Molecular immunology of antibodies and $\mathbf{T}$ cell receptors}

\author{
T H RABBITTS
}

\begin{abstract}
Antigen receptors
The key feature of the immune system is the ability to react and neutralise foreign substances, known as antigens, when they invade the body. These antigens-for example, viral coat proteinsare removed by the immune system primarily by two types of white cells (lymphocytes) circulating in the blood: these are the B lymphocytes and T lymphocytes (called B cells and T cells respectively). Because of the large number of potential foreign antigens the immune system has developed a highly variable set of antigen receptors, which are found on the surface of $B$ and $T$ cells. Each cell expresses only one receptor, and the complete repertoire is provided by the large number of different $B$ and $T$ cells available at any one time. The $B$ and $T$ cell receptors are related in their structure, but important differences distinguish B cell receptors
\end{abstract}

Medical Research Council Laboratory of Molecular Biology, Cambridge CB2 2QH

T H RABBITTS, BSC, PHD, member of scientific staff (the antibodies) and T cell receptors. Perhaps the main feature is the ability of antibodies to be secreted into the plasma by the subfraction of B cells concerned with combating a particular infection. Secretion of $\mathrm{T}$ cell receptors does not appear to occur.

$\mathrm{T}$ cell receptors are associated with other proteins on the cell surface which seem to have a vital role in the function of the cell. One such molecule is a membrane protein called CD4 (formerly T4), which is a characteristic of a subset of T cells which help B cells to produce antibody. This so called helper $T$ cell population is the primary target for the virus which causes the acquired immune deficiency syndrome (AIDS), and the CD4 molecule appears to serve as a recognition site for the virus itself-that is, it is the receptor on T cells for the AIDS virus. Thus during the development of AIDS there is a progressive loss of helper $\mathrm{T}$ cells and associated loss of ability to produce secreted antibodies, the key feature of antibody mediated responses.

Antibodies and $\mathrm{T}$ cell receptors are found on the outside of the cell surface of $B$ and $T$ cells respectively. The proteins are attached to the cell surface by a so called constant region; the part of the protein which fixes on to the antigen is the variable region, which is free on the outside of the cell. The latter name is explicit since the differences between individual receptors on the vast population of lymphocytes are found in this part of the molecule. The functions of the receptor, such as generating signals to tell the cell to start 
multiplying after association with antigen, are encoded by the constant region.

The basic cellular immunology of a response to an infection is fairly straightforward. The $B$ and $T$ cells mature from bone marrow cells, and in doing so place on their surface an antigen receptor. The antigen receptor derives from an extraordinary set of gene rearrangements (which will be described below), but the main fact that allows successful functioning of the immune system is that one cell expresses one receptor. These receptors have the ability to recognise and bind to one antigen only, thus providing the fine specificity of the system. Once the specific $B$ and $T$ cells have found and interacted with the foreign antigen they divide rapidly, producing a large number of cells carrying the specific receptor for the infective agent. In this way the infection can be neutralised.

\section{Antigen receptor genes}

How are the unique receptors created in each of the vast number of B and T cells? The answer is simple but mechanically complicated. All cells in the body possess genes coding for antibodies and $T$ cell receptors, but these are organised as a set of small separate chromosomal segments which only in $B$ and $T$ cells undergo assortment, creating unique combinations. In this way only $B$ and $T$ cells end up with receptors, and, furthermore, the variability of the receptor is created.

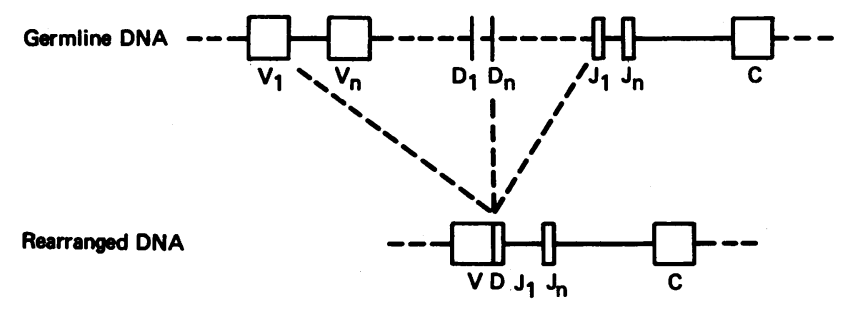

mRNA

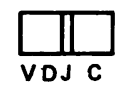

Protein

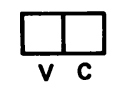

Examples so far:

$$
\begin{array}{ll}
\text { Immunoglobulin (antibody) } & -H, L(K, \lambda) \\
T \text { cell receptor } & -\alpha, \beta \\
& -\gamma
\end{array}
$$

Composition of rearranging genes. DNA=deoxyribonucleic acid; $m R N A=$ messenger ribonucleic acid; $V=$ variable segment; $D=$ diversity segment; $\mathrm{J}=$ joining segment; $\mathrm{C}=$ constant segment.

The precise composition of the receptor genes is as follows. There are several segments in each antigen receptor gene locus: variable, diversity, joining, and constant segments. The progenitors of the cells that become mature $B$ or $T$ cells have the capacity to rearrange their chromosomes so that individual variable, diversity, and joining segments are joined to create a specific, complete receptor gene. $B$ cells have an antibody heavy chain gene and a light chain gene functionally rearranged, and these give rise to the antibody molecule. Similarly, in $T$ cells an $\alpha$ chain and a $\beta$ chain are rearranged to give rise to the $T$ cell receptor. The figure shows how all antigen receptor genes are inherited and rearranged in the maturation of this immune response.

Clearly, the number of combinations which can be achieved for a fully joined variable region depends on the number of subunits concerned and the number of individual segments within each subunit. Variable regions in receptors are made by a combination of inherited variable segments, diversity segments, and joining segments. Thus if there are 100 variable segments, 10 diversity segments, and 5 joining segments it is possible to generate $100 \times 10 \times 5$ different combinations-that is, 5000 distinct combinations of variable regions.

Variability in antibodies can also be augmented by a process called somatic mutation. This is a process whereby changes are introduced into the antibody gene while it is being assembled and after it is assembled. These changes are apparently randomly introduced and are therefore specific to each cell which is assembling antibody genes. $T$ cell receptor genes do not appear to have this facility.

Both antibody and $T$ cell receptor genes have a more subtle way of adding to variability during gene assembly. This entails changes of sequence artificiaily introduced at the junctions joining variable to diversity and diversity to joining segments. Major sequence changes can occur by this process and add a further major change in overall sequence (which varies from cell to cell). The consequences of the assembly of the receptor gene and the associated changes which occur is that each cell ends up with a unique active receptor. The individual therefore possesses a set of circulating lympoid cells which are ready to inactivate infections as they occur.

\section{Immune deficiency syndromes}

There are a number of immune deficiency syndromes which result in the inability of afflicted individuals to mount antibody responses. The well known $\mathrm{X}$ linked agammaglobulinaemia is an inherited disorder in which males who inherit the defective $X$ chromosome cannot elicit antibody responses or do so ineffectively. An analogous disorder is late onset agammaglobulinaemia, in which individuals acquire the immunodeficiency-that is, there is apparently no inherited aspect.

Recently, it has also become clear that antigen receptor genes are, inadvertently, concerned with the development of specific lymphoid tumours by facilitating major chromosomal abnormalities. A well documented example of this phenomenon is seen in the B cell tumour Burkitt's lymphoma. All tumours of this type have a specific chromosomal translocation in which pieces of two distinct chromosomes have been exchanged. One of the affected chromosomes carries an antibody gene, and molecular studies have shown that the chromosomal break occurs immediately next to this gene. The critical feature for tumour development is the other chromosome concerned in the abnormality. This chromosome carries a proto-oncogene which is close to the junction of the translocation. (Proto-oncogenes, which are discussed in a previous article in this series, are so called because they can contribute to tumour formation when they become activated-for example, by mutation: thus the transition from proto-oncogene (a gene with normal cellular activity) to oncogene (a gene with abnormal activity) can take place by relatively small changes to the gene.) Since in Burkitt's lymphoma a proto-oncogene is relocated to the proximity of the antibody gene after the translocation this "activates" it into an oncogene, contributing to the transformation of the B cell. It is probably not coincidence that receptor genes may be implicated in the process of chromosomal translocation. Since these genes are themselves rearranging for normal receptor formation it is likely that the translocation is a mistake, which has drastic consequences. This view is strongly supported by observations of antibody genes in translocations of other B cell tumours-for example, follicular lymphoma-and of $\mathrm{T}$ cell receptor genes in chromosomal abnormalities of acute and chronic $T$ cell leukaemia.

In conclusion, the ability of receptor genes to assemble themselves from small pieces in normal lymphoid cell development is clearly important for survival of the individual in facilitating immune recognition. There seems to be a price to pay, however, in that mistakes in the normal receptor rearrangements (which entail damage to proto-oncogenes) can result in lymphoid tumour induction. 